\title{
Development of a Chirality-Sensitive Flexibility
}

\section{Descriptor for 3+3D-QSAR}

\author{
Máté Dervarics, ${ }^{a}$ Ferenc Ötvös ${ }^{b}$ and Tamás A. Martinek $*^{* a}$
}

${ }^{a}$ Institute of Pharmaceutical Chemistry, University of Szeged, H-6701 Szeged, POB 121, Hungary and ${ }^{\mathrm{b}}$ Institute of Biochemistry, Biological Research Center of the Hungarian Academy of Sciences, H-6726 Szeged, Temesvári krt 62, Hungary

EMAIL: martinek@ pharm.u-szeged.hu

TITLE RUNNING HEAD: Chirality Sensitive Flexibility Descriptor

Table S1. The best PLS models optimized by a Monte Carlo Annealing protocol in the descriptor space

Figure S1. Dependence of the endomorphin $\mathrm{L} 50 \% \mathrm{O} \mathrm{q}^{2}$ values on the rejection factor utilized for filtering out descriptors from PPQs where the Pearson correlogram calculated for PPQ point-plane distance distributions showed multiple peaks. 3

Figure S2. Probability distribution of a chance correlation by using PLS for endomorphin analogs.

Figure S3. Relative importance (PLS coeff * stddev) of the descriptors calculated from the PLS model without MCA for endomorphin analogs.

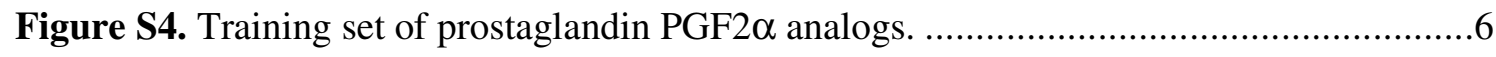

Figure S5. Dependence of the prostaglandin L50\% $\mathrm{O} \mathrm{q}^{2}$ values on the rejection factor utilized for filtering out descriptors from PPQs where the Pearson correlogram calculated for PPQ point-plane distance distributions showed multiple peaks.

Figure S6. Probability distribution of a chance correlation by using PLS for prostaglandin analogs.

Figure S7. Relative importance (PLS coeff * stddev) of the descriptors calculated from the PLS model without MCA for prostaglandin analogs. 
Table S1. The best PLS models optimized by a Monte Carlo Annealing protocol in the descriptor space

\begin{tabular}{|c|c|c|c|c|c|}
\hline $\begin{array}{c}\text { Model \#1 } \\
\text { Descriptors }\end{array}$ & PLS coeff. & $\begin{array}{c}\text { Model \#2 } \\
\text { Descriptors }\end{array}$ & PLS coeff. & $\begin{array}{c}\text { Model \#3 } \\
\text { Descriptors }\end{array}$ & PLS coeff. \\
\hline b_a c $h$ & 1.6506 & $b a c h$ & 1.4879 & $b a c h$ & 1.9692 \\
\hline b_a_d_h & 0.9283 & b_a_d_h & 0.5925 & d_f_h_i & 1.4901 \\
\hline d_f_h_i & 1.4519 & d_f_h_i & 1.6826 & Constant & 11.6021 \\
\hline Constant & 14.8839 & Constant & 13.5173 & & \\
\hline rank & 2 & rank & 2 & rank & 2 \\
\hline$r^{2}$ & 0.74 & $r^{2}$ & 0.73 & $r^{2}$ & 0.70 \\
\hline LOO-q ${ }^{2}$ & 0.69 & LOO- $q^{2}$ & 0.67 & LOO- $q^{2}$ & 0.65 \\
\hline L50\%O- $q^{2}$ & 0.65 & L50\%O- $q^{2}$ & 0.63 & L50\%O- $q^{2}$ & 0.62 \\
\hline
\end{tabular}




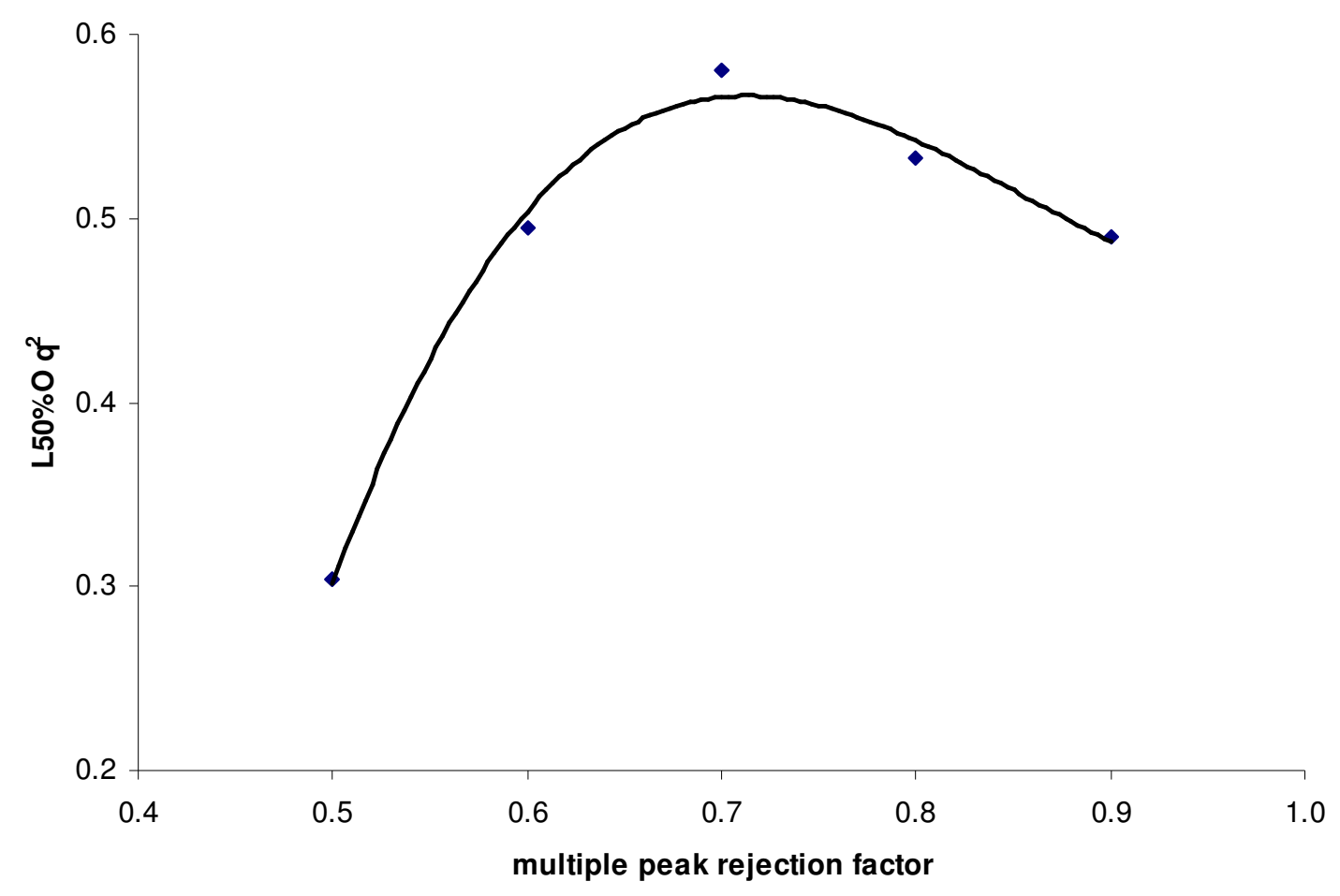

Figure S1. Dependence of the endomorphin L50\%O q $\mathrm{q}^{2}$ values on the rejection factor utilized for filtering out descriptors from PPQs where the Pearson correlogram calculated for PPQ point-plane distance distributions showed multiple peaks.

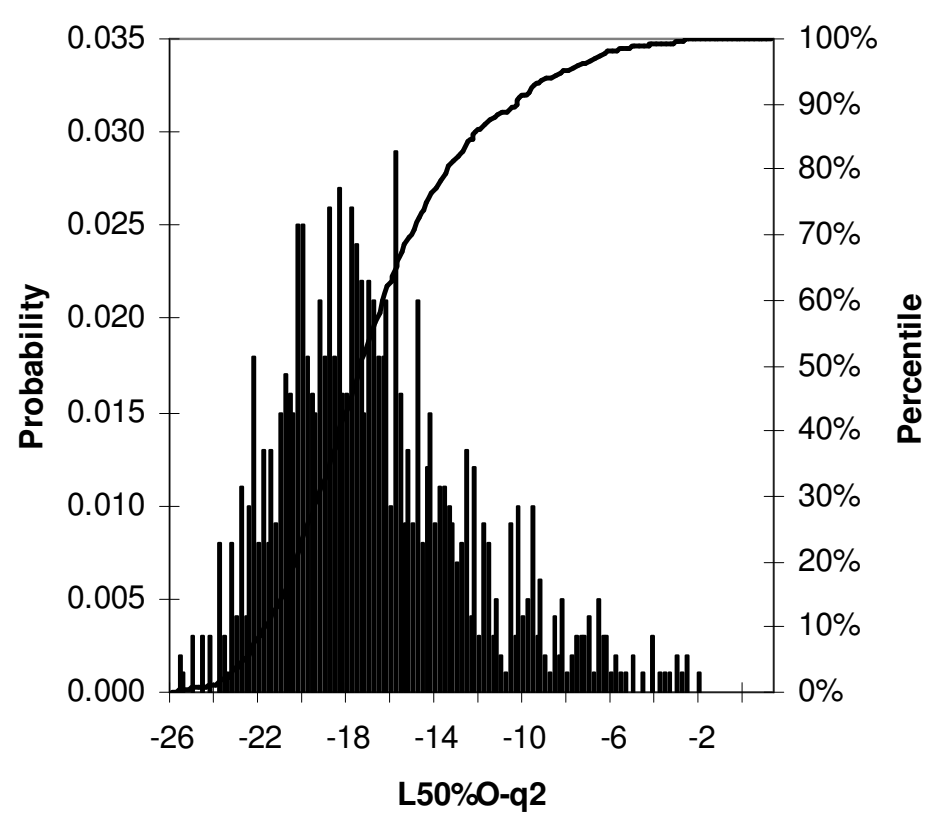

Figure S2. Probability distribution of a chance correlation by using PLS for endomorphin analogs. 


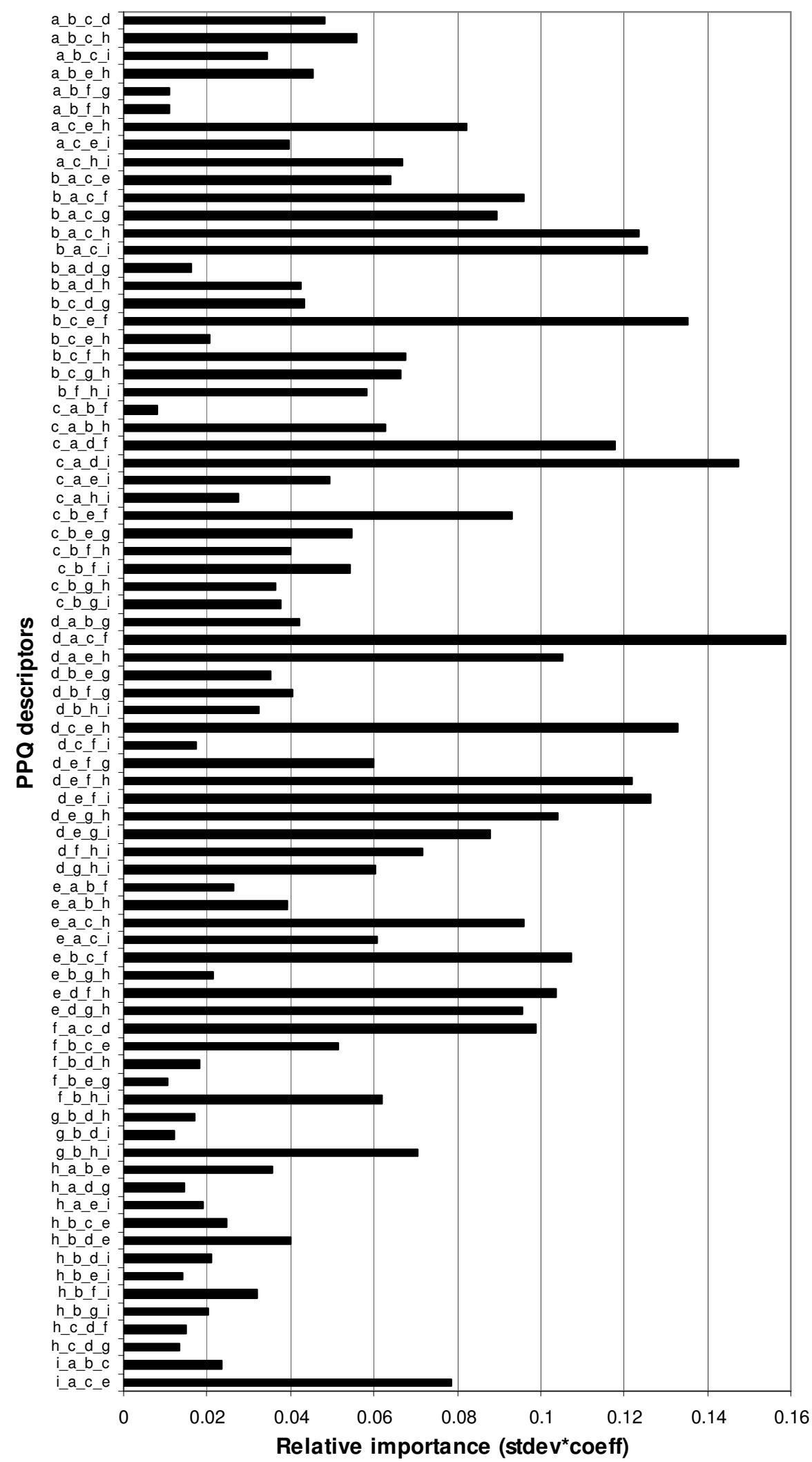

Figure S3. Relative importance (PLS coeff * stddev) of the descriptors calculated from the PLS model without MCA for endomorphin analogs. 

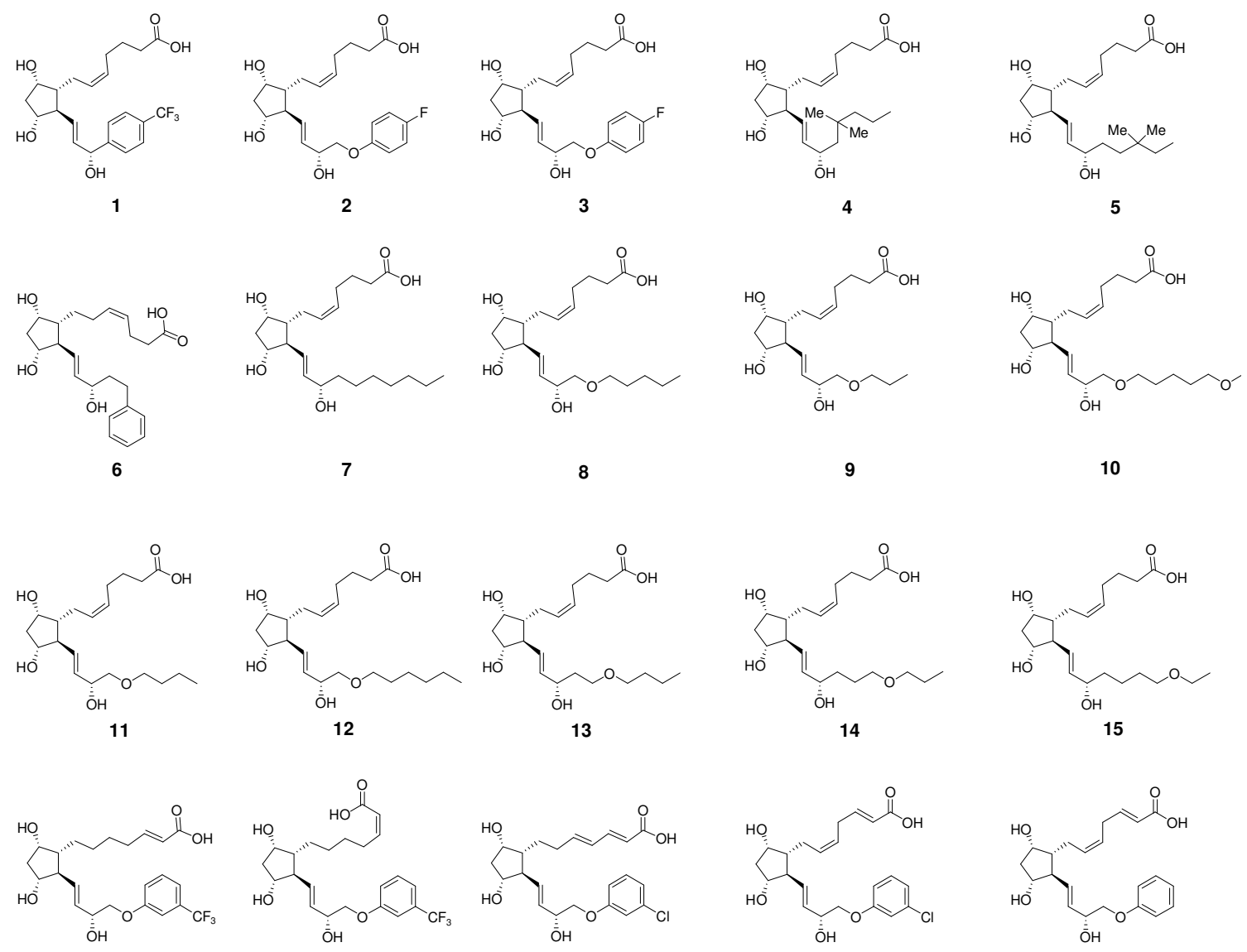

16

17

18

19

20
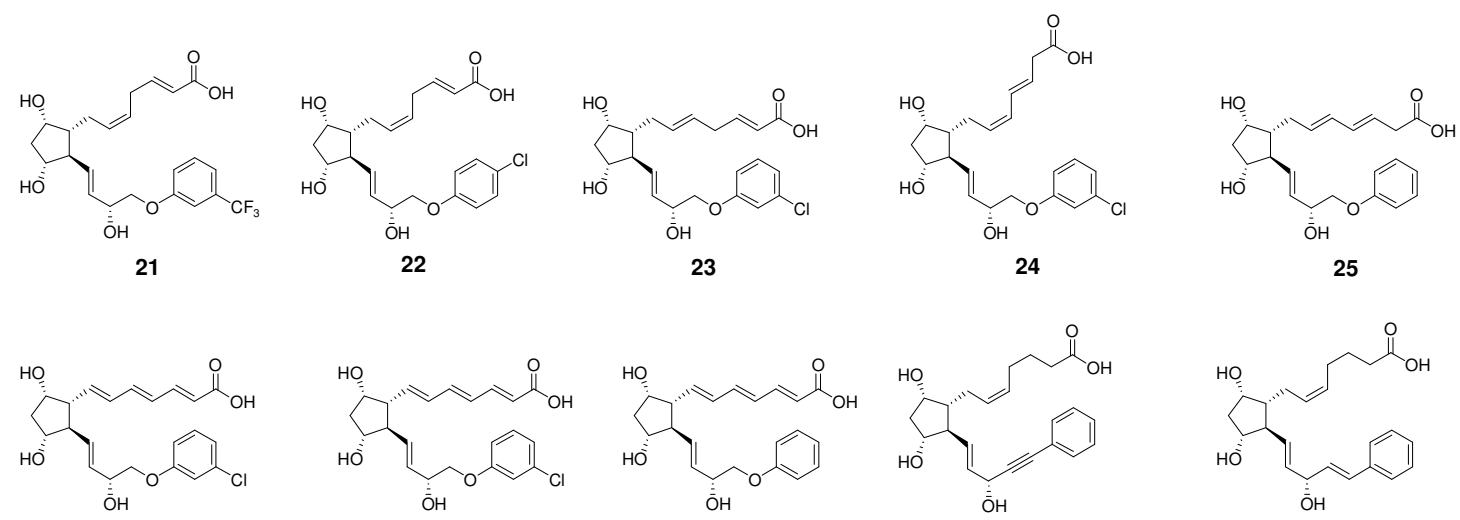

26

28

29

30 

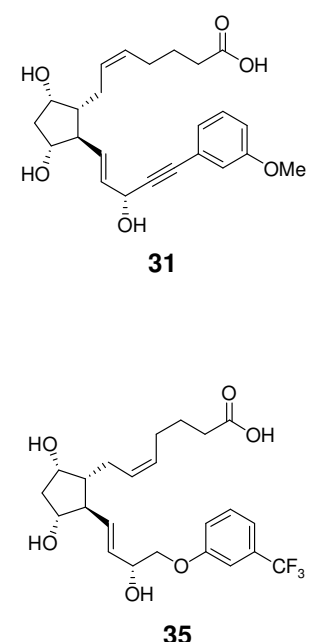
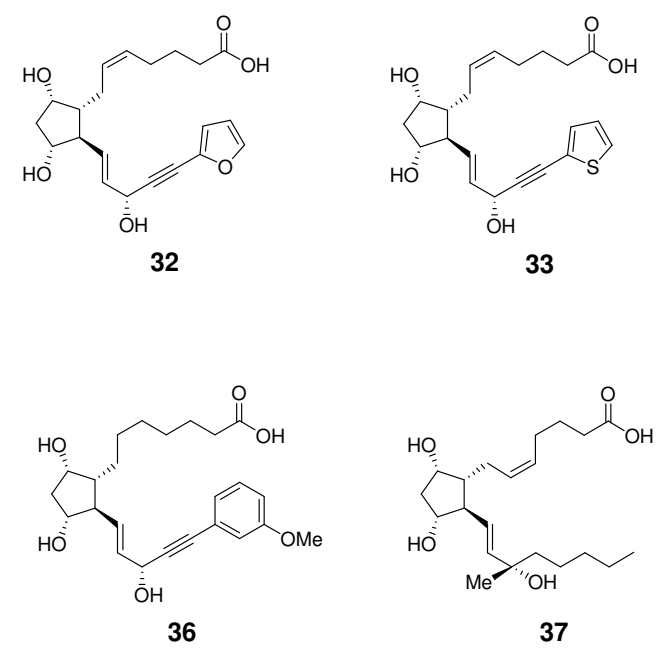

Figure S4. Training set of prostaglandin PGF2 $\alpha$ analogs.
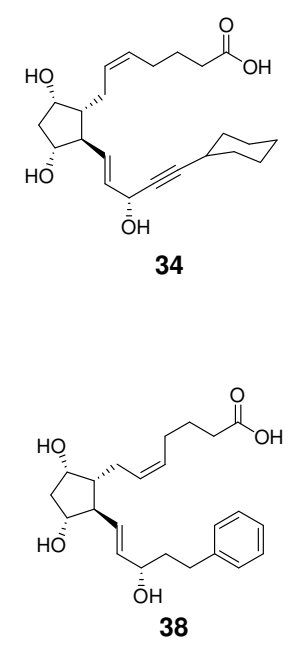


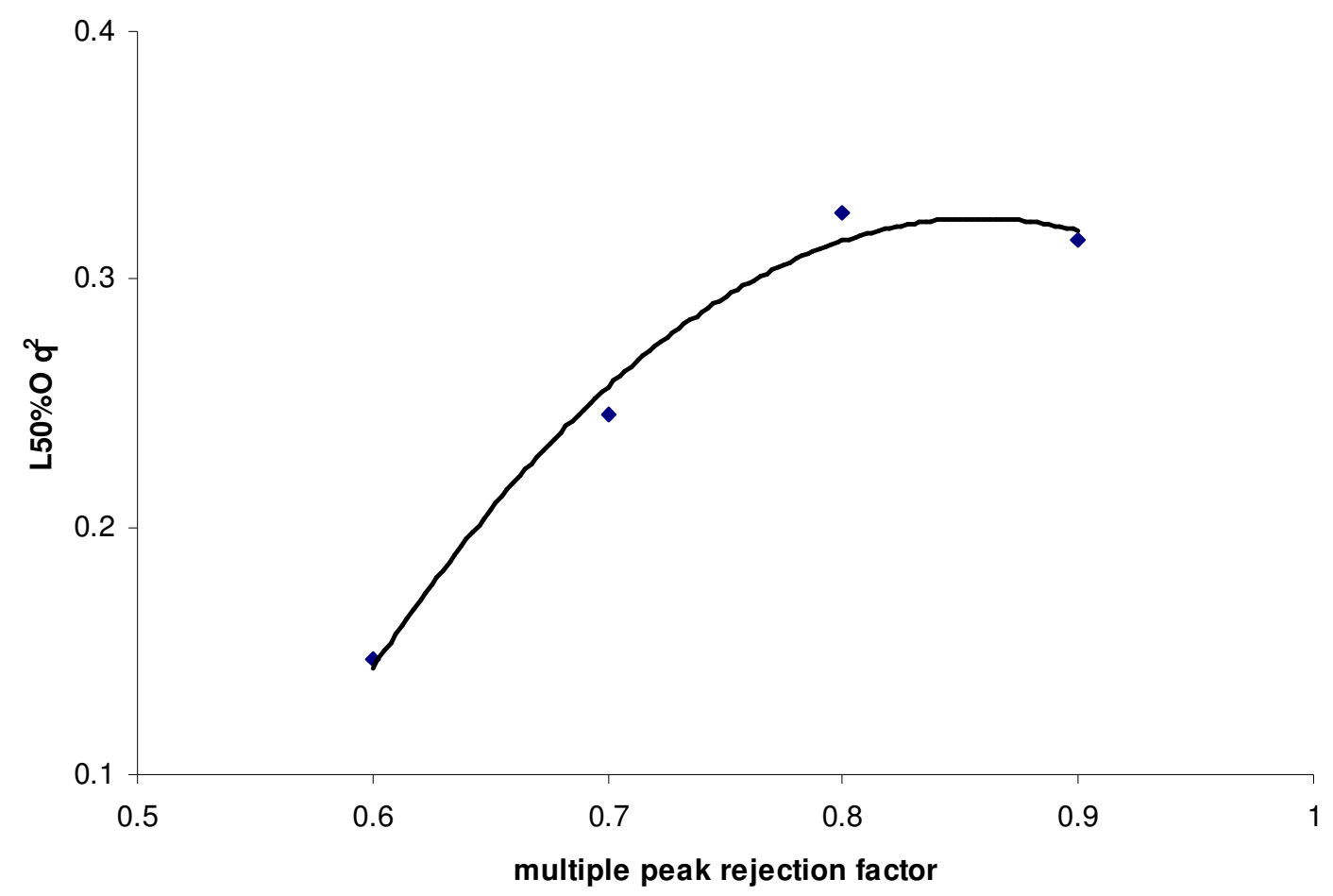

Figure S5. Dependence of the prostaglandin L50\%O q $\mathrm{q}^{2}$ values on the rejection factor utilized for filtering out descriptors from PPQs where the Pearson correlogram calculated for PPQ point-plane distance distributions showed multiple peaks. 


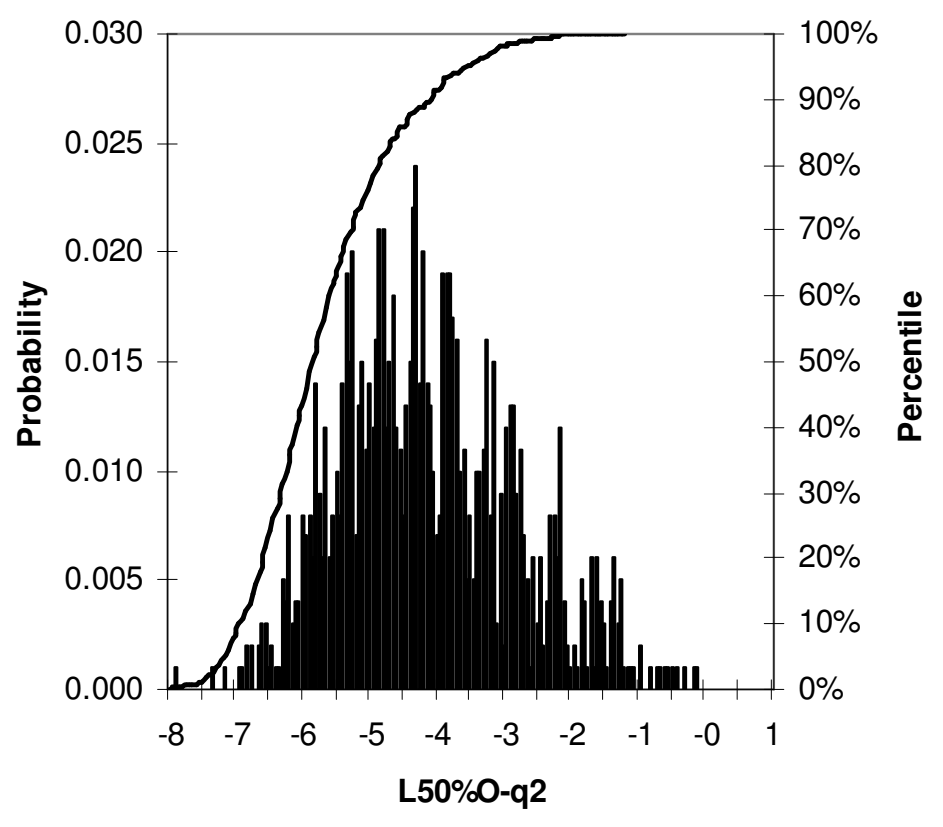

Figure S6. Probability distribution of a chance correlation by using PLS for prostaglandin analogs. 


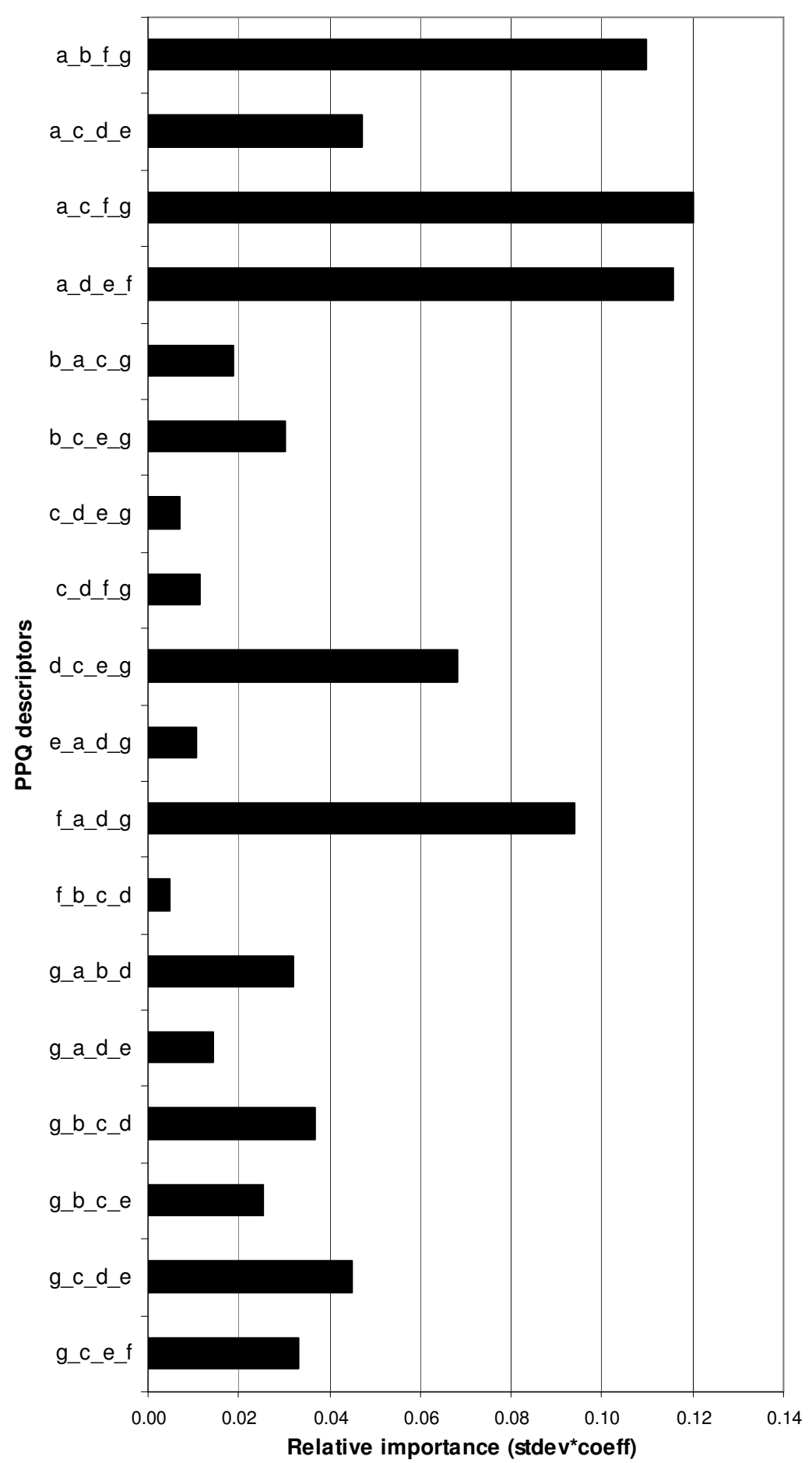

Figure S7. Relative importance (PLS coeff * stddev) of the descriptors calculated from the PLS model without MCA for prostaglandin analogs. 